\title{
Does sleep deprivation alter functional EEG networks in children with focal epilepsy?
}

\author{
Eric van Diessen ${ }^{1 *}$, Willem M. Otte ${ }^{1,2}$, Kees P. J. Braun ${ }^{1}$, Cornelis J. Stam ${ }^{3}$ and Floor E. Jansen ${ }^{1}$ \\ ${ }^{1}$ Department of Pediatric Neurology, Brain Center Rudolf Magnus, University Medical Center Utrecht, Utrecht, Netherlands \\ ${ }^{2}$ Biomedical MR Imaging and Spectroscopy Group, Image Sciences Institute, University Medical Center Utrecht, Utrecht, Netherlands \\ ${ }^{3}$ Department of Clinical Neurophysiology, VU University Medical Center, Amsterdam, Netherlands
}

\section{Edited by:}

Linda Douw, Martinos Center for

Biomedical Imaging, USA

Reviewed by:

Raffaele Ferri, Oasi Institute for

Research on Mental retardation and

Brain Aging, Italy

Steven Marshall Stufflebeam,

Harvard Medical School, USA

\section{*Correspondence:}

Eric van Diessen, Department of

Pediatric Neurology, University

Medical Center Utrecht,

KC 03.063.0, PO Box 85090,

3508 AB Utrecht, Netherlands

e-mail: e.vandiessen-3@

umcutrecht.nl

\begin{abstract}
Electroencephalography (EEG) recordings after sleep deprivation increase the diagnostic yield in patients suspected of epilepsy if the routine EEG remains inconclusive. Sleep deprivation is associated with increased interictal EEG abnormalities in patients with epilepsy, but the exact mechanism is unknown. In this feasibility study, we used a network analytical approach to provide novel insights into this clinical observation. The aim was to characterize the effect of sleep deprivation on the interictal functional network organization using a unique dataset of paired routine and sleep deprivation recordings in patients and controls. We included 21 children referred to the first seizure clinic of our center with suspected new onset focal epilepsy in whom a routine interictal and a sleep deprivation EEG (SD-EEG) were performed. Seventeen children, in whom the diagnosis of epilepsy was excluded, served as controls. For both time points weighted functional networks were constructed based on interictal artifact free time-series. Routine and sleep deprivation networks were characterized at different frequency bands using minimum spanning tree (MST) measures (leaf number and diameter) and classical measures of integration (path length) and segregation (clustering coefficient). A significant interaction was found for leaf number and diameter between patients and controls after sleep deprivation: patients showed a shift toward a more path-like MST network whereas controls showed a shift toward a more star-like MST network. This shift in network organization after sleep deprivation in patients is in accordance with previous studies showing a more regular network organization in the ictal state and might relate to the increased epileptiform abnormalities found in patients after sleep deprivation. Larger studies are needed to verify these results. Finally, MST measures were more sensitive in detecting network changes as compared to the classical measures of integration and segregation.
\end{abstract}

Keywords: epilepsy, EEG, sleep deprivation, network analysis, graph theory, minimum spanning tree

\section{INTRODUCTION}

An interictal electroencephalogram (EEG) is routinely acquired in patients suspected of epilepsy to support the clinical diagnosis. Nevertheless, the interictal EEG recording often lacks epileptiform abnormalities or is insufficient to determine classification of the epilepsy syndrome. EEG recordings after sleep deprivation (SD-EEG) improve the diagnostic yield of interictal EEG recordings (Malow, 2004; Smith, 2005; Wirrell, 2010). In the past, debate has centered on the question whether it is the recording of sleep itself or sleep deprivation that promotes the increased presence of epileptiform abnormalities and thereby its sensitivity. Converging evidence exists that SD-EEG recordings improve the detection of epileptiform abnormalities and help to determine classification of the epilepsy syndrome, independently of the presence of sleep during the EEG recording (Ellingson et al., 1984; Fountain et al., 1998).

The mechanism for the increased presence of epileptiform abnormalities after sleep deprivation, however, remains unclear. There is converging evidence that sleep and sleep deprivation causes variability in cortical excitability that might be related to the increased presence of epileptiform abnormalities, for review see (Badawy et al., 2012). Another, possible complementary, explanation for this clinical phenomenon might originate from an altered functional network organization after sleep deprivation (Koenis et al., 2013). Brain functioning is increasingly perceived as a complex network of interacting brain regions (Bassett and Bullmore, 2009; Bullmore and Sporns, 2009; Stam and Van Straaten, 2012), which can be studied using, among others, EEG recordings. Quantification of connections between brain regions enables characterization of functional network organization by means of network analysis. Network analysis reduces complex systems, such as the brain, to a collection of nodes (brain regions) and edges (connections between brain areas). From these networks several measures can be inferred to characterize global changes and efficiency in network organization: the clustering coefficient (a measure of segregation) and path length (a measure of integration). Normal brain functioning relies on an adequate balance of local segregation and global integration (Bullmore and Sporns, 2009; Stam, 2010). Koenis et al. showed that network organization 
changes in healthy subjects after sleep deprivation toward a more integrated and less segregated network (Koenis et al., 2013). Interestingly, functional networks in epilepsy are repeatedly identified as "less efficient" as this organization changes in the ictal state toward a more segregated and less integrated network (Van Diessen et al., 2013). We hypothesized that sleep deprivation would change the interictal network toward a more segregated and less integrated network, deviant from healthy controls.

Network analytical studies require various choices and assumptions that influence the outcome (Van Wijk et al., 2010). For example, it remains unclear how differences in network density (i.e., number of connections) should be taken into account when comparing networks between groups. A solution for this problem might be offered by the so-called minimum spanning tree (MST) approach. The MST approach creates a unique network based on the weighted connections between nodes. Furthermore, it connects all the nodes in the network without forming cycles and thereby reducing the connection costs. In this way, networks are obtained with an identical number of connections and thereby facilitating comparison between groups by taking only the most important connections into account. An increasing number of neuropsychiatric studies have already successfully used this approach to characterize networks and to improve group comparisons (Lee et al., 2006; Ortega et al., 2008; Schoen et al., 2011; Boersma et al., 2013). To investigate an added value of the MST approach in understanding the increased presence of epileptogenic abnormalities after sleep deprivation, we compared the results of traditional weighted network measures (clustering coefficient and path length) with MST network measures.

\section{MATERIALS AND METHODS PATIENTS}

Children who visited the outpatient First Seizure Clinic of the University Medical Center Utrecht, the Netherlands, between January 2006 and December 2012 were eligible for our study. We included children in whom both a routine EEG and SDEEG recording was performed for clinical reasons. We accepted a maximum interval of 9 months between routine EEG and SD-EEG since aging is associated with alterations in functional network organization (Boersma et al., 2011). Children in whom the diagnosis of focal epilepsy was definite-as judged by the occurrence of multiple seizures, their semiology, and supported by neurophysiological recordings or neuroimaging-were compared to a control group. The control group consisted of children who were referred with clinical events suspected of seizures but in whom the diagnosis of epilepsy was eventually excluded, based on expert opinion, on clinical follow-up, and on the results of ancillary investigations, including routine and SD-EEG recordings. For both groups, we excluded children with neurological or psychiatric co-morbidities, including the presence of developmental delay of unknown origin. The institutional ethical committee approved the study and concluded that the Dutch Medical Research Involving Human Subjects Act did not apply, and written informed consent was not required.

\section{DATA ACQUISITION AND SELECTION}

The EEG was recorded at 21 scalp electrodes (Fp1, Fp2, F8, F4, Fz, F3, F7, A2, T8, C4, Cz, C3, T7, A1, P8, P4, Pz, P3, P7, O1, and $\mathrm{O} 2$ ), according to the international 10-20 system (SystemPlus Evolution, Micromed), against G2 as a reference electrode (placed between $\mathrm{Cz}$ and $\mathrm{Fz}$ ) and referenced to an average montage for further analysis. Impedance of each electrode was kept below $5 \mathrm{k} \Omega$. Data was high- and low-pass filtered at 0.5 and $70 \mathrm{~Hz}$, respectively. The sampling frequency was $512 \mathrm{~Hz}$.

All EEG recordings were acquired for clinical purposes. Therefore, the recorded segments suitable for selection of resting state EEG epochs, were limited in their length to 2-3 min. During these segment recordings children were awake and had their eyes-closed. From these we selected four epochs of $8.19 \mathrm{~s}$ (each containing 4096 samples). Epochs were visually inspected by one of the authors [EvD]. The minimal number of epochs was based on a previous network study that acquired stable network characteristics within patients using four epochs of similar length (Douw et al., 2013). In order to investigate the actual contribution of sleep deprivation on functional networks, the epochs from SD-EEG recordings were selected before sleep and drowsiness, when an occipital alpha rhythm was still present and slow eye-movements were absent. Epochs were chosen free of epileptiform abnormalities, abnormal slowing and electrocardiographic or motion-induced artifacts, for which two frontoparietal and basal temporal electrodes (Fp1, Fp2, A1, and A2) were excluded. Furthermore, all epochs were selected at the beginning of the EEG recordings from EEG recordings that were conducted in the morning to increase homogeneity of our data. The epochs were independently re-inspected by a clinical epileptologist [Floor E. Jansen] on artifacts and epileptiform abnormalities. Finally, the selected EEG epochs were converted to ASCII files for further analysis.

\section{EVALUATION OF ADDED CLINICAL VALUE SLEEP DEPRIVATION EEG}

EEG recordings after sleep deprivation were performed to improve the diagnostic yield. We considered an SD-EEG of added clinical value when the SD-EEG recording provided: (1) new information regarding localization of the epileptogenic focus and (2) additional information regarding spreading of epileptiform activity. A confirmation of the clinical findings of the first EEG recording was not considered as added value.

\section{FUNCTIONAL CONNECTIVITY}

Communication between brain areas in EEG networks can be quantified using functional connectivity. The level to which different brain areas are functionally connected depends on the level of synchronous temporal activity, irrespective of signal amplitude (Varela et al., 2001). Although computational differences exist between various functional connectivity measures (Pereda et al., 2005), all of these measures assume that an increase in linear and nonlinear correlations of frequency specific neurophysiologic activity between two brain areas-also referred to as "synchronization"-is associated with an increased communication between both brain areas (Bassett and Bullmore, 2009; Bullmore and Sporns, 2009; Stam and Van Straaten, 2012). 
In this study, functional connectivity between electrodes was computed from each selected epoch by means of the Phase Lag Index (PLI) (Stam et al., 2007) [Brainwave software; http:// home.kpn.nl/stam7883/brainwave.html version 0.9 .116 authored by (Cornelis J. Stam)]. A PLI value for each pair of electrodes was computed for each epoch and the average PLI (over four epochs) was used for further analysis. The PLI was calculated separately for the following frequency bands: delta $(0.5-4 \mathrm{~Hz})$, theta $(4-8 \mathrm{~Hz})$, alpha $(8-13 \mathrm{~Hz})$, and beta $(13-30 \mathrm{~Hz})$. The PLI is a measure that is less sensitive for confounding than other functional connectivity measures and quantifies the phase coupling between two time series as a value between 0 and 1 (Stam et al., 2007). The synchronization between time series is based on the consistency of the nonzero phase lag of one time series with the other. To compute the instantaneous phase difference for each time sample we used the analytical signal concept and the Hilbert transform. During the calculation the influence of volume conduction is diminished by disregarding phase differences of zero. A PLI value of 0 indicates no phase coupling between signals, or coupling with a phase difference centered on $0 \pm \pi$ radians. A PLI $>0$ indicates the presence of phase coupling. An index of the asymmetry of the phase difference distribution can be obtained from a time series of phase differences $\Delta \Phi\left(t_{k}\right)$ in the following way: $P L I=\left|\left\langle\operatorname{sign}\left[\Delta \Phi\left(t_{k}\right)\right]\right\rangle\right|$ where $\Delta \Phi$ is the difference between instantaneous phases for two time-series $[-\pi$, $\pi], t_{k}$ are discrete steps, and in which $<>$ denotes the average over time $t$. A more detailed description of the calculation and specifications of PLI can be found elsewhere (Stam et al., 2007).

\section{NETWORK ANALYTICAL MEASURES}

\section{Network construction}

For each dataset, we constructed a weighted undirected network, described by the graph $G=(N, W)$, where $N$ is the set of all 17 EEG electrodes and $W=\left\{w_{i j}\right\}$ is the $N \times N$ symmetric weight matrix, where $w_{i i}=0$ and $w_{i j}$ the PLI determined between node $i$ and $j$. Global network properties were quantified via weighted clustering coefficient and path length. We repeated the construction of functional networks and analysis for different frequency ranges.

\section{Weighted clustering coefficient}

The clustering coefficient is a measure of degree to which nodes in a graph tend to cluster together. We used the weighted clustering coefficient $\mathrm{C}$ as described in Grindrod (2002), Higham et al. (2007), Stam et al. (2009). To calculate C from weighted networks, the weights between node $i$ and other nodes $j$ should be symmetrical $\left(w_{i j}=w_{j i}\right)$ and $0 \leq w_{i j}=1$. These conditions are fulfilled when using PLI as weight definition. The (weighted) $\mathrm{C}$ of node $i$ is then defined as

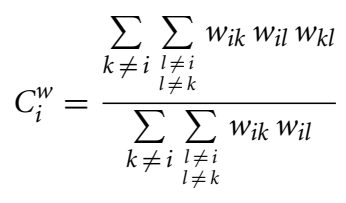

in the sums of this formula $i=k, i=l$, and $k=l$ are not included. In the case in which $w_{i j}$ equals either 0 or 1 the definition was equivalent to the definition for unweighted graphs (Watts and Strogatz, 1998). The mean weighted clustering coefficient was defined as

$$
C_{\text {mean }}^{w}=\frac{1}{N} \sum_{i=1}^{N} C_{i}^{w}
$$

\section{Weighted path length}

for a given node $i$ in the graph, the path algorithm finds the path with lowest cost (i.e., the shortest path length) between that node and every other node. For the weighted path length, the path between two nodes $i$ and $j$ is found by minimizing the sum of weights assigned to the edges on their path. The average path length (L) for node $i$ to all other nodes is defined as

$$
L_{i}^{w}=\frac{1}{N-1} \sum_{i \neq j}^{N} \min \left\{l_{i j}^{w}\right\}
$$

Here, $\min \left\{l_{i j}^{w}\right\}$ is the weighted path length between node $i$ and $j$, calculated using Dijkstra's algorithm (Dijkstra, 1959). We considered high values of the PLI as close functional distance and low values of the synchronization index as large functional distance (i.e., $l_{i j}^{w}=1 / w_{i j}$ ). In our dataset no disconnected nodes were present. The mean weighted path was defined as

$$
L_{\text {mean }}^{w}=\frac{1}{N} \sum_{i=1}^{N} l_{i}^{w}
$$

\section{Normalization}

for each functional dataset, path length and clustering coefficient were normalized using 50 surrogate networks as previously described in Stam et al. (2009). This number was sufficient to result in stable surrogate network properties (defined as less than $1 \%$ variability in network parameters such as path length and clustering coefficient). Normalized weighted path length was defined as $L /\left\langle L_{\text {surrogate }}\right\rangle$ and normalized clustering coefficient was defined as $C /\left\langle C_{\text {surrogate }}\right\rangle$.

\section{MINIMUM SPANNING TREE}

The MST creates a unique network based on the weighted connections between nodes. Furthermore, it connects all the nodes in the network without forming cycles and thereby reduces the connection costs. To compute the MST we used Kruskal's algorithm (Kruskal, 1956). This algorithm orders the weight of all edges in an ascending way, and subsequently starts the construction of a MST performing the following step as many times as possible: adding the edge with the highest PLI until all nodes $N$ are connected in a loopless network consisting of $N-1$ edges. Thus, if adding an edge results in formation of a cycle within the network, this edge will be skipped. Given the number of electrodes included in our analysis, trees contained 17 nodes and 16 edges. From these trees, several measures can be quantified (Boersma et al., 2013). For our study, we focused on the two most straightforward MST measures: leaf number and diameter that give information on topological features of trees (Figure 2). The leaf number is defined by the number of nodes in the tree with degree $=1$ 
(i.e., these nodes are connected by only one edge to the network) and has a lower bound of 2 and an upper bound of $\mathrm{m}=N-1$. The leaf number presents an upper bound to the diameter of the spanning tree that is the largest distance between any possible pair of nodes of the tree. The upper limit of the diameter of the tree is defined as diameter $=\mathrm{m}-$ leaf number +2 , implying that the largest possible diameter will decrease with increasing leaf number (Boersma et al., 2013). Both leaf number and diameter were normalized between 0 and 1 . In summary, by excluding less important connections in the network, the MST network is built from the most efficient connections and enables a direct comparison between two networks since the number of nodes and connections are similar (Figure 1).

\section{STATISTICAL ANALYSIS}

First, we explored the effect of sleep deprivation in each group separately by comparing relative power spectra, network and MST measures from routine EEG and SD-EEG recordings for each frequency band with a paired $t$-test. To investigate whether a different network alteration was observed after sleep deprivation in patients compared with controls, we used a repeated measures analysis of variance (ANOVA) with type of EEG (EEG vs. SDEEG) as within factor and group (patients vs. controls) as between factor and type of EEG $\times$ group as interaction for each frequency band. Differences between groups in age and gender were explored using independent $t$-test and chi-square test respectively. To correct for multiple comparison, we used false discovery rate correction per frequency band as each frequency band is associated with distinct network and functions (Basar et al., 2001). All analyses were performed in SPSS. A p-value below 0.05 was considered significant.

\section{RESULTS}

\section{PATIENT CHARACTERISTICS}

In total, 21 children with a definitive diagnosis of focal epilepsy were included in this study (5 girls, mean age $10.7 \pm 3.1$ years).
Clinical details are summarized in Table 1. Average time between EEG and SD-EEG recording was $2.4 \pm 2.6$ months. The control group consisted of seventeen children ( 7 girls, mean age $10.5 \pm 2.5$ years). Average time between their EEG and SD-EEG recording was $1.9 \pm 1.1$ months. No significant differences were found for age $(t=-0.11, p=0.9)$ and gender $\left(\chi^{2}=1.3, p=\right.$ $0.3)$ between patient and control groups. In three patients, antiepileptic drug treatment was initiated after routine EEG recording and before SD-EEG recording. In 15 out of 21 patients (71\%) the SD-EEG provided new information regarding localization of the epileptic focus or on the spreading of epileptiform activity and therefore considered of additional clinical value (Table 1). No epileptiform activity was found in controls during SD-EEG recording.

\section{DIFFERENCES BETWEEN REGULAR AND SD-EEG IN RELATIVE POWER SPECTRA PER GROUP}

Paired $t$-tests were performed for relative power spectra per frequency band for patients and controls separately. In patients, no significant differences between spectra obtained from standard EEGs and SD-EEGs were found (Table 2). In controls, however, an increase of the relative spectrum in the beta frequency band was found after sleep deprivation $(p=0.012)$.

\section{DIFFERENCES BETWEEN REGULAR AND SD-EEG IN NETWORK AND MST MEASURES PER GROUP}

Paired $t$-tests were performed for each network and MST characteristic per frequency band for patients and controls separately (Tables 3A,B respectively). In patients, a significant decrease in leaf number $(p=0.041)$ after sleep deprivation was found only in the alpha frequency band. In the other frequency bands, no significant differences in network measures were found after sleep deprivation. In controls, the clustering coefficient ( $p=$ $0.021)$ and leaf number $(p=0.014)$ in the delta frequency band increased after sleep deprivation and the diameter decreased $(p=0.037)$.

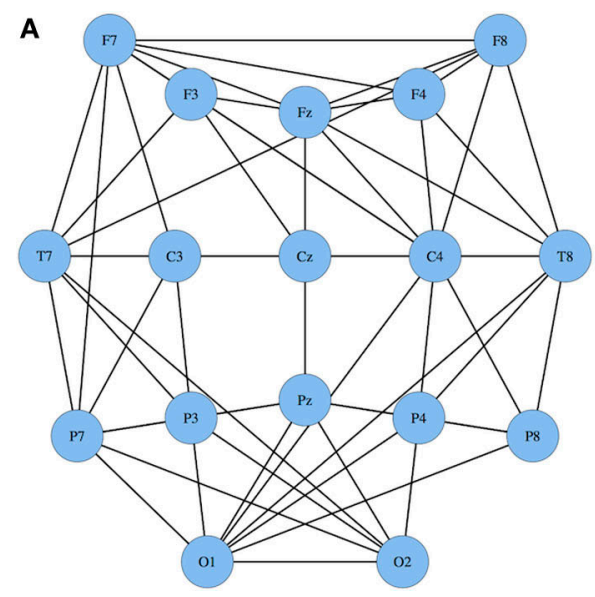

FIGURE 1 | Two schematic illustrations of networks. (A) a standard network and (B) a MST network wherein all nodes are connected only once resulting in a loopless network. In panel (B)

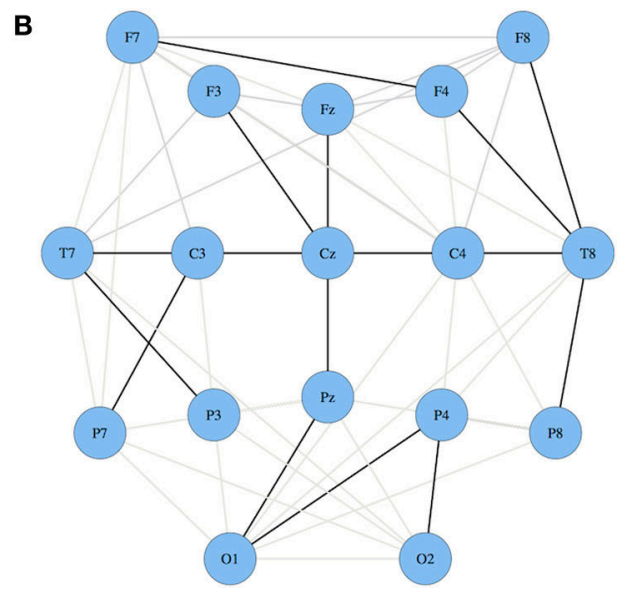

the black lines represent the most efficient connections in the MST network; grey lines represent the excluded functional connections. 


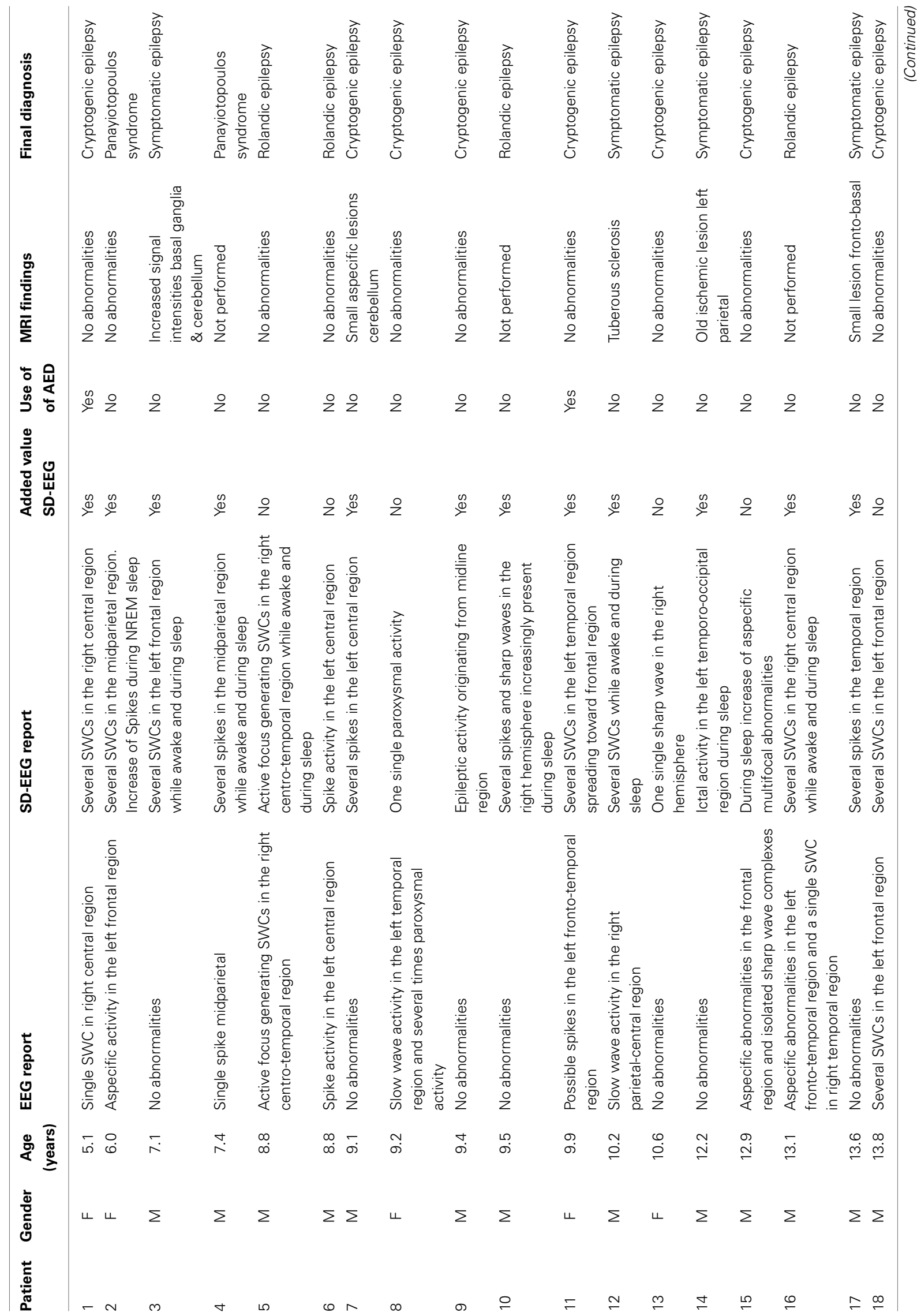




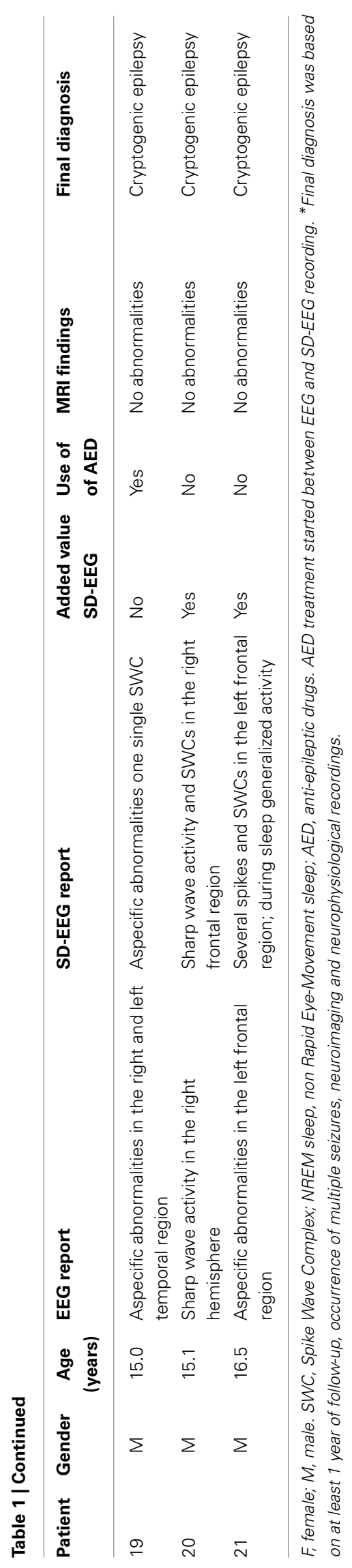

\section{INTERACTION IN NETWORK AND MST MEASURES}

Repeated measures ANOVA were performed for each network and MST characteristic separately for each frequency band. Significant interaction was only found for the leaf number $(p=$ $0.03)$ and a trend for diameter $(p=0.061)$ in the alpha frequency band (Table 4). The interaction for leaf number and diameter increased when including only patients in whom SD-EEG recording was considered to be of additional diagnostic value $(p=0.009$ and $p=0.03$ respectively) (Figure 3 ).

\section{DISCUSSION}

An EEG after sleep deprivation is often performed in patients suspected of epilepsy when the standard EEG recording is inconclusive. Little is known, however, about the mechanism behind the increased sensitivity of SD-EEG recordings in epilepsy. In this study we investigated whether a network analytical approach could clarify this phenomenon. Our repeated measures analysis for leaf number and diameter suggested an interaction between patients and controls after sleep deprivation: patients showed a shift toward a more path-like MST network whereas controls showed a shift toward a more star-like MST network (Figure 2). This shift difference was more pronounced when including only the patients in whom the SD-EEG recording was of additional clinical value (Table 4). Together with the increased presence of epileptiform abnormalities after sleep deprivation in patients, the network shift toward a more path-like MST network could possibly reflect an inadequate compensatory mechanism of the epileptic brain, although this requires to be confirmed using data from future studies with larger sample sizes.

Previous research has shown that functional networks change during seizure generation and propagation into a more regular network organization (i.e., highly segregated and poorly integrated network) (Ponten et al., 2007; Schindler et al., 2007; Kramer et al., 2008, 2010; Schindler et al., 2008; Ponten et al., 2009). This shift toward a regular topology might depend on the decreased centrality of so-called "hub-nodes" in the network or an altered synchronizability during the ictal state (Van Diessen et al., 2013). Furthermore, long-term continuous evaluation of functional networks derived from intracranial recordings, revealed large fluctuations in clustering coefficient and path length during the day (Kuhnert et al., 2010). These fluctuations over time, largely attributed to daily rhythms, showed an increased regularization of functional networks during nighttime in patients with focal epilepsy (Kuhnert et al., 2010) whereas a shift toward a more optimal segregation and integration of the network during sleep was observed in healthy subjects (Ferri et al., 2008). Possibly, this shift toward a more regular network during sleep in patients with epilepsy explains why the epileptic brain is more susceptible to both epileptiform discharges and seizures during sleep. However, this remains speculative as we cannot infer a causal relation between network alteration and an increased presence of interepileptic discharges after sleep deprivation based on our results. We did not investigate network alterations during sleep, but a similar mechanism could explain the increased presence of epileptiform discharges after sleep deprivation. This study suggests that the network organization shift toward a more path-like topology in patients with 
Table 2 | Paired $t$-test between EEG and SD-EEG recordings (for patients and controls separately) for relative power spectra in five frequency bands.

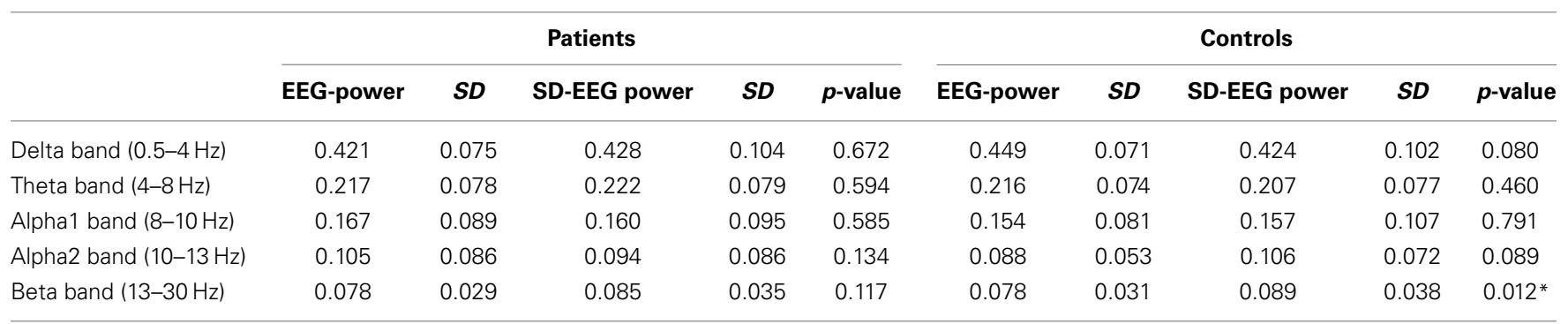

Alpha band is separated in a lower and upper frequency band. SD, standard deviation. ${ }^{*}$ Significant $(p<0.05)$.

Table 3A | Paired $t$-test between EEG and SD-EEG recordings of patients for network measures (path length and clustering coefficient) and MST measures (diameter and leaf number).

\begin{tabular}{|c|c|c|c|c|c|c|c|c|}
\hline & \multicolumn{2}{|c|}{ Delta band $(0.5-4 \mathrm{~Hz})$} & \multicolumn{2}{|c|}{ Theta band $(4-8 \mathrm{~Hz})$} & \multicolumn{2}{|c|}{ Alpha band $(8-13 \mathrm{~Hz})$} & \multicolumn{2}{|c|}{ Beta band $(13-30 \mathrm{~Hz})$} \\
\hline Clustering coefficient & 0.317 & 0.755 & -1.840 & 0.081 & -1.803 & 0.086 & 1.304 & 0.207 \\
\hline Diameter & 0.383 & 0.705 & 0.447 & 0.660 & 1.366 & 0.187 & -0.923 & 0.367 \\
\hline Leaf number & 1.072 & 0.297 & -0.103 & 0.916 & -2.189 & $0.041^{*}$ & 1.769 & 0.092 \\
\hline
\end{tabular}

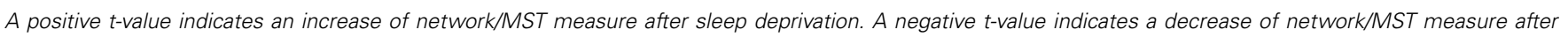
sleep deprivation. * Significant $(p<0.05)$.

Table 3B | Paired $t$-test between EEG and SD-EEG recordings of controls for network measures (path length and clustering coefficient) and MST measures (diameter and leaf number).

\begin{tabular}{|c|c|c|c|c|c|c|c|c|}
\hline & \multicolumn{2}{|c|}{ Delta band $(0.5-4 \mathrm{~Hz})$} & \multicolumn{2}{|c|}{ Theta band $(4-8 \mathrm{~Hz})$} & \multicolumn{2}{|c|}{ Alpha band $(8-13 \mathrm{~Hz})$} & \multicolumn{2}{|c|}{ Beta band $(13-30 \mathrm{~Hz})$} \\
\hline Clustering coefficient & 2.561 & $0.021 * *$ & -0.449 & 0.659 & 0.770 & 0.452 & -0.687 & 0.502 \\
\hline Diameter & -2.269 & $0.037^{* *}$ & 0.438 & 0.668 & -1.409 & 0.178 & -0.682 & 0.505 \\
\hline Leaf number & 2.759 & $0.014 * *$ & -0.502 & 0.623 & 1.007 & 0.329 & -0.054 & 0.958 \\
\hline
\end{tabular}

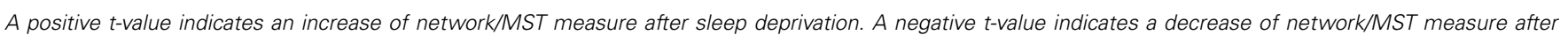
sleep deprivation. * Significant $(p<0.05),{ }^{* *}$ Significant after correcting for multiple post-hoc comparisons (false discovery rate test).

epilepsy (i.e., increased diameter and decreased leaf number). Considering the path-like network as a network wherein nodes are less centrally connected and share basic characteristics with a regular network, it might be possible that the mechanisms underlying sleep deprivation-induced network alterations mimic the changes of a functional network during the ictal state or during sleep. Interestingly, these changes in network organization could coincide with an increase of cortical excitability that has previously been described as a pro-convulsive effect, accountable for the induction of epileptogenic activity after sleep deprivation (Badawy et al., 2006; Manganotti et al., 2006). As mentioned previously, functional connectivity in EEG networks is based on the assumption that an increased synchronization of frequency specific neurophysiologic activity implies an improved communication between brain areas. The change toward a more path-like network in patients with focal epilepsy after sleep deprivation suggests an increased synchronization of spatially closely related brain areas. The underlying mechanism behind the increased synchronization could indeed be an increased cortical excitability, as repeatedly shown by studies combining high-frequency transcranial magnetic stimulation and EEG connectivity measures in healthy controls, particularly in the alpha frequency band (Fuggetta et al., 2008; Plewnia et al., 2008). To which extent this relation is also accountable for epilepsy remains speculative until further investigations will simultaneously map cortical excitability and associated changes in function network organization. Furthermore, we found the largest group difference in the alpha frequency band. Previous studies have suggested that each frequency band is associated with distinct networks and cognitive processes (Von Stein and Sarnthein, 2000; Basar et al., 2001). For example, spontaneous alpha activity is typically found when eyes are closed whereas task-related alpha activity is 
Table 4 | Interactions from repeated measures ANOVA for each network and MST measures.

\begin{tabular}{|c|c|c|c|c|}
\hline & \multirow{2}{*}{\multicolumn{2}{|c|}{$\begin{array}{c}\begin{array}{c}\text { Interaction(all } \\
\text { patients, } n=21 \text { ) }\end{array} \\
\text { Type of EEG* group }\end{array}$}} & \multirow{2}{*}{\multicolumn{2}{|c|}{$\begin{array}{c}\text { Interaction (added value } \\
\text { SD-EEG, } n=15 \text { ) } \\
\text { Type of EEG }{ }^{*} \text { group }\end{array}$}} \\
\hline & & & & \\
\hline & $F$-value & $p$-value & $F$-value & $p$-value \\
\hline \multicolumn{5}{|c|}{ DELTA-BAND $(0.5-4 \mathrm{~Hz})$} \\
\hline Path length & 0.115 & 0.737 & 0.008 & 0.928 \\
\hline Clustering coefficient & 2.123 & 0.154 & 0.456 & 0.505 \\
\hline Diameter & 3.976 & 0.054 & 2.256 & 0.144 \\
\hline Leaf number & 2.144 & 0.152 & 1.359 & 0.253 \\
\hline \multicolumn{5}{|l|}{ THETA-BAND (4-8 Hz) } \\
\hline Path length & 1.018 & 0.320 & 1.395 & 0.247 \\
\hline Clustering coefficient & 0.394 & 0.534 & 0.321 & 0.575 \\
\hline Diameter & 1.558 & 0.220 & 0.183 & 0.672 \\
\hline Leaf number & 0.103 & 0.750 & 0.361 & 0.552 \\
\hline \multicolumn{5}{|c|}{ ALPHA-BAND $(8-13 \mathrm{~Hz})$} \\
\hline Path length & 0.075 & 0.785 & 0.059 & 0.809 \\
\hline Clustering coefficient & 2.881 & 0.098 & 2.038 & 0.164 \\
\hline Diameter & 3.735 & 0.061 & 4.724 & $0.038^{*}$ \\
\hline Leaf number & 5.101 & $0.030^{*}$ & 7.680 & $0.009 * *$ \\
\hline \multicolumn{5}{|l|}{ BETA-BAND $(13-30 \mathrm{~Hz})$} \\
\hline Path length & 0.977 & 0.330 & 0.216 & 0.645 \\
\hline Clustering coefficient & 1.852 & 0.182 & 0.077 & 0.783 \\
\hline Diameter & 0.058 & 0.812 & 0.030 & 0.864 \\
\hline Leaf number & 1.360 & 0.251 & 1.539 & 0.224 \\
\hline
\end{tabular}

In the left column, the interactions when including all patients $(n=21)$. In the right column, the interactions when including only those patients in whom the SD-EEG was of added clinical value $(n=15)$. * Significant $(p<0.05)$, ** Significant after correcting for multiple post-hoc comparisons (false discovery rate test).

typically found during sensory, motor and primarily top-down cognitive processes (Von Stein and Sarnthein, 2000). Although it remains uncertain how our results relate to changes in the cognitive domain, they suggest an involvement of the alpha frequency band in normal sleep physiology. However, the exact relationship remains an open question.

The MST approach has been suggested as an appropriate method to overcome certain limitations of network studies, particularly in relation to differences in network densities between groups (Van Wijk et al., 2010). Nevertheless, few studies have compared standard network measures, such as clustering coefficient and path length, to MST measures (Boersma et al., 2013). We found particular network alteration between controls and patients for the MST measures leaf number and diameter, suggesting that these measures are perhaps more sensitive to network alterations in patients with epilepsy. Larger studies investigating and comparing both approaches are needed to verify this. In addition, like clustering coefficient and path length, leaf number and diameter are highly correlated, arguing for more and distinct MST features to characterize networks.

To date, only one study has investigated the influence of sleep deprivation on functional networks (Koenis et al., 2013), but only in healthy subjects. Koenis and others reported network

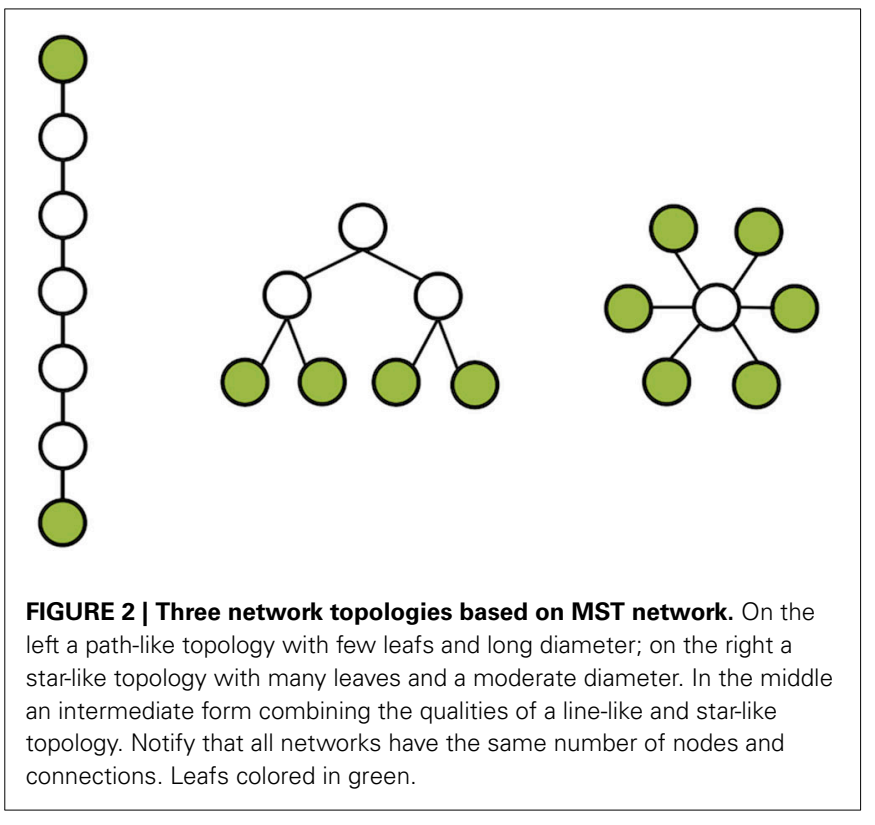

alterations in the alpha frequency band during an eyes-closed condition, namely a shift toward a more random network. In our control group, we found an increase in clustering coefficient, a decreased diameter and increased leaf number in the delta frequency band. Together, these results suggest a more star-like MST network in controls after sleep deprivation. Interestingly, a star-like MST network shares basic characteristics with that of a random network organization (i.e., a poorly segregated and highly integrated network). Although we found a similar shift in network organization as Koenis and others, it is challenging to explain the differences in frequency bands between both studies (alpha vs. delta). Possibly, a different patient population (age) and methodology (different EEG recordings) are accountable for these differences (Van Wijk et al., 2010; Smit et al., 2012). Besides network measures, we also measured the relative power spectrum. In accordance with previous literature, we found an increase in beta power spectrum after sleep deprivation in healthy subjects (Gast et al., 2011) whereas we did not find this effect in patients. Possibly, the lack of increased beta power in patients after sleep deprivation reflects an inadequate compensation of the epileptic brain to maintain beta frequency band specific functions, such as integration of multi-sensory information (Von Stein and Sarnthein, 2000).

Although our study provides further insight in the mechanism of increased sensitivity of EEG recordings after sleep deprivation, several limitations should be mentioned. First, our patient sample is limited ( 21 children) and included only children with focal epilepsy. One could therefore argue that our results might be restricted to this age range and type of patients. Nevertheless, the increased sensitivity of SD-EEG recordings is particularly manifested in children suspected of focal epilepsy (Shinnar et al., 1994) and our population is therefore ideal to study this phenomenon. Secondly, the control group contained children who were initially suspected of having suffered from an epileptic seizure. Although epilepsy was excluded after clinical 


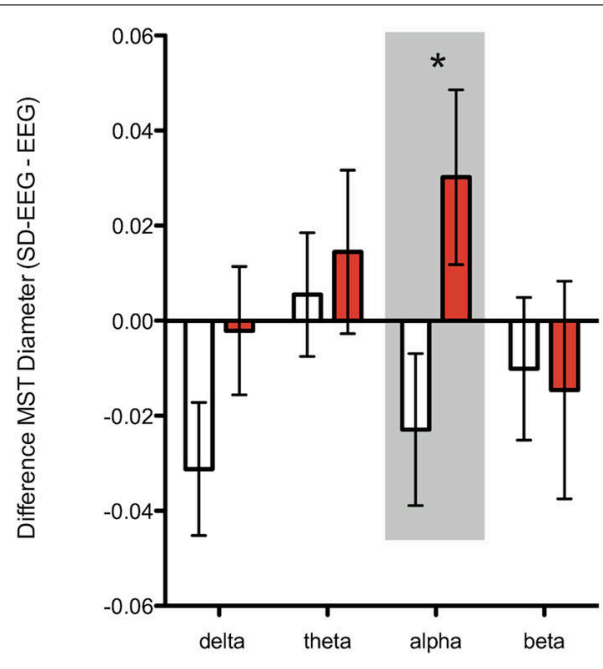

FIGURE 3 | Illustration of interaction effects from MST measures diameter (left graph) and leaf number (right graph) per frequency band as revealed with a repeated measures ANOVA (mean values and standard error of the mean bars). In this analysis we included only patients in whom the SD-EEG was of added value $(n=15)$, and all controls $(n=17)$ (Table 4). There was a significant interaction for diameter in the alpha band; the diameter increased in patients whereas an opposite

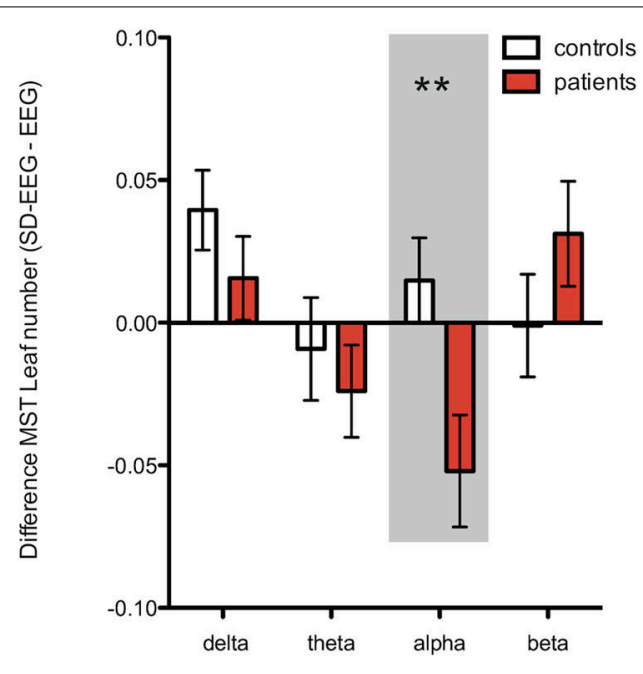

effect was found for controls. For leaf number, a significant interaction was found in the alpha band; the leaf number decreased in patients whereas an opposite effect was found for controls. Together, these results in the alpha band suggest a shift toward a path-like topology for patients after sleep deprivation and a shift toward a star-like topology for controls.

*Significant $(p<0.05)$, **Significant after correcting for multiple post-hoc comparisons (false discovery rate test). evaluation and follow-up, this could have introduced a bias in our results. As compared to truly healthy children who never experienced any paroxysmal event, the network organization of our control group could be altered as well. As a consequence this might have reduced our statistical group differences. Otherwise, it would be difficult to receive ethical approval for performing SDEEG recordings in a healthy pediatric population. Furthermore, since we used clinical EEG recordings, there was only a limited fraction of EEG recording available from which we could select resting state epochs. Based on previous research, we assume that four epochs is enough to ensure stable network measures (Douw et al., 2013). The availability of more epochs would allow further exploration on the variability - and thus stability-of these network measures by, for instance, performing a leaveone-out analysis. Unfortunately, in this study we did not have sufficient data to do so, but we suggest future studies to characterize this variability using long-term EEG recordings. In three patients anti-epileptic drugs were started before the SD-EEG recording (patients 1,11, and 19). Considering the potential influence of anti-epileptic drugs on functional networks (Van Diessen et al., 2013), this could have deviated our results. In all three patients, however, treatment started only a few days before SD-EEG recording and was therefore still at a very low dosage. An additional analysis, after excluding these patients, revealed similar results. Finally, our sample size is small and the FDR-correction to minimize inflation of the type I error might not be perfect. Additional validation is therefore needed to confirm our findings. Despite these limitations, we believe that our results legitimate a larger network study wherein the mechanism of increased sensitivity of EEG recordings after sleep deprivation will be investigated more thoroughly. To enable a subanalysis of different epilepsy syndromes and allow correlation analysis between network measures and clinical characteristics such as age, gender and use of anti-epileptic drugs a larger study cohort is required.

In conclusion, this study provides insights into the mechanisms behind the increased presence of epileptiform abnormalities after sleep deprivation in children with focal epilepsy. We suggest that an inadequate compensatory shift of the epileptic network toward a more path-like topology after sleep deprivation is accountable for the increased epileptiform abnormalities often found in patients with epilepsy.

\section{AUTHOR CONTRIBUTIONS}

Designed the experiments: Eric van Diessen, Willem M. Otte, Kees P. J. Braun, Cornelis J. Stam and Floor E. Jansen. Performed the experiments: Eric van Diessen, Willem M. Otte. Wrote the graph analysis software: Cornelis J. Stam. Analyzed the data: Eric van Diessen, Willem M. Otte. Wrote the paper: Eric van Diessen, Willem M. Otte, Kees P. J. Braun, Cornelis J. Stam and Floor E. Jansen.

\section{FUNDING}

Eric van Diessen, Willem M. Otte, and Kees P. J. Braun are supported by the Dutch National Epilepsy Fund (NEF 09-93, NEF 12-05 and NEF 08-10, respectively).

\section{REFERENCES}

Badawy, R. A., Curatolo, J. M., Newton, M., Berkovic, S. F., and Macdonell, R. A. (2006). Sleep deprivation increases cortical excitability in epilepsy: syndrome-specific effects. Neurology 67, 1018-1022. doi: 10.1212/01.wnl.0000237392.64230.f7

Badawy, R. A., Freestone, D. R., Lai, A., and Cook, M. J. (2012). Epilepsy: ever-changing states of cortical excitability. Neuroscience 222, 89-99. doi: 10.1016/j.neuroscience.2012.07.015 
Basar, E., Basar-Eroglu, C., Karakas, S., and Schurmann, M. (2001). Gamma, alpha, delta, and theta oscillations govern cognitive processes. Int. J. Psychophysiol. 39, 241-248. doi: 10.1016/S0167-8760(00)00145-8

Bassett, D. S., and Bullmore, E. T. (2009). Human brain networks in health and disease. Curr. Opin. Neurol. 22, 340. doi: 10.1097/WCO.0b013e32 832d93dd

Boersma, M., Smit, D. J., Boomsma, D. I., De Geus, E. J., Delemarre-Van De Waal, H. A., and Stam, C. J. (2013). Growing trees in child brains: graph theoretical analysis of electroencephalography-derived minimum spanning tree in 5and 7-year-old children reflects brain maturation. Brain Connect. 3, 50-60. doi: 10.1089/brain.2012.0106

Boersma, M., Smit, D. J., De Bie, H. M., Van Baal, G. C., Boomsma, D. I., De Geus, E. J., et al. (2011). Network analysis of resting state EEG in the developing young brain: structure comes with maturation. Hum. Brain Mapp. 32, 413-425. doi: $10.1002 / \mathrm{hbm} .21030$

Bullmore, E., and Sporns, O. (2009). Complex brain networks: graph theoretical analysis of structural and functional systems. Nat. Rev. Neurosci. 10, 186-198. doi: $10.1038 / n r n 2575$

Dijkstra, E. W. (1959). A note on two problems in connexion with graphs. Numerische mathematik 1, 269-271. doi: 10.1007/BF01386390

Douw, L., De Groot, M., Van Dellen, E., Aronica, E., Heimans, J. J., Klein, M., et al. (2013). Local MEG networks: the missing link between protein expression and epilepsy in glioma patients? Neuroimage 75C, 203-211. doi: 10.1016/j.neuroimage.2013.02.067

Ellingson, R. J., Wilken, K., and Bennett, D. R. (1984). Efficacy of sleep deprivation as an activation procedure in epilepsy patients. J. Clin. Neurophysiol. 1, 83-101. doi: 10.1097/00004691-198401000-00005

Ferri, R., Rundo, F., Bruni, O., Terzano, M. G., and Stam, C. J. (2008). The functional connectivity of different EEG bands moves towards small-world network organization during sleep. Clin. Neurophysiol. 119, 2026-2036. doi: 10.1016/j.clinph.2008.04.294

Fountain, N. B., Kim, J. S., and Lee, S. I. (1998). Sleep deprivation activates epileptiform discharges independent of the activating effects of sleep. J. Clin. Neurophysiol. 15, 69-75. doi: 10.1097/00004691-199801000-00009

Fuggetta, G., Pavone, E. F., Fiaschi, A., and Manganotti, P. (2008). Acute modulation of cortical oscillatory activities during short trains of high-frequency repetitive transcranial magnetic stimulation of the human motor cortex: a combined EEG and TMS study. Hum. Brain Mapp. 29, 1-13. doi: 10.1002/hbm. 20371

Gast, H., Schindler, K., Rummel, C., Herrmann, U. S., Roth, C., Hess, C. W., et al. (2011). EEG correlation and power during maintenance of wakefulness test after sleep-deprivation. Clin. Neurophysiol. 122, 2025-2031. doi: 10.1016/j.clinph.2011.03.003

Grindrod, P. (2002). Range-dependent random graphs and their application to modeling large small-world Proteome datasets. Phys. Rev. E Stat. Nonlin. Soft Matter Phys. 66:066702. doi: 10.1103/PhysRevE.66.066702

Higham, D. J., Kalna, G., and Vass, J. K. (2007). Spectral analysis of twosigned microarray expression data. Math. Med. Biol. 24, 131-148. doi: 10.1093/imammb/dql030

Koenis, M. M., Romeijn, N., Piantoni, G., Verweij, I., Van Der Werf, Y. D., Van Someren, E. J., et al. (2013). Does sleep restore the topology of functional brain networks? Hum. Brain Mapp. 34, 487-500. doi: 10.1002/hbm.21455

Kramer, M. A., Eden, U. T., Kolaczyk, E. D., Zepeda, R., Eskandar, E. N., and Cash, S. S. (2010). Coalescence and fragmentation of cortical networks during focal seizures. J. Neurosci. 30, 10076-10085. doi: 10.1523/JNEUROSCI.6309-09.2010

Kramer, M. A., Kolaczyk, E. D., and Kirsch, H. E. (2008). Emergent network topology at seizure onset in humans. Epilepsy. Res. 79, 173-186. doi: 10.1016/j.eplepsyres.2008.02.002

Kruskal, J. B. Jr. (1956). On the shortest spanning subtree of a graph and the traveling salesman problem. Proc. Am. Math. Soc. 7, 48-50. doi: 10.1090/S00029939-1956-0078686-7

Kuhnert, M. T., Elger, C. E., and Lehnertz, K. (2010). Long-term variability of global statistical properties of epileptic brain networks. Chaos 20, 043126. doi: $10.1063 / 1.3504998$

Lee, U., Kim, S., and Jung, K. Y. (2006). Classification of epilepsy types through global network analysis of scalp electroencephalograms. Phys. Rev. E Stat. Nonlin. Soft Matter Phys. 73:041920. doi: 10.1103/PhysRevE.73.041920

Malow, B. A. (2004). Sleep deprivation and epilepsy. Epilepsy. Curr. 4, 193-195. doi: 10.1111/j.1535-7597.2004.04509.x
Manganotti, P., Bongiovanni, L. G., Fuggetta, G., Zanette, G., and Fiaschi, A. (2006). Effects of sleep deprivation on cortical excitability in patients affected by juvenile myoclonic epilepsy: a combined transcranial magnetic stimulation and EEG study. J. Neurol. Neurosurg. Psychiatry 77, 56-60. doi: 10.1136/jnnp.2004.041137

Ortega, G. J., Sola, R. G., and Pastor, J. (2008). Complex network analysis of human ECoG data. Neurosci. Lett. 447, 129-133. doi: 10.1016/j.neulet.2008. 09.080

Pereda, E., Quiroga, R. Q., and Bhattacharya, J. (2005). Nonlinear multivariate analysis of neurophysiological signals. Prog. Neurobiol. 77, 1-37. doi: 10.1016/j.pneurobio.2005.10.003

Plewnia, C., Rilk, A. J., Soekadar, S. R., Arfeller, C., Huber, H. S., Sauseng, P., et al. (2008). Enhancement of long-range EEG coherence by synchronous bifocal transcranial magnetic stimulation. Eur. J. Neurosci. 27, 1577-1583. doi: 10.1111/j.1460-9568.2008.06124.x

Ponten, S. C., Bartolomei, F., and Stam, C. J. (2007). Small-world networks and epilepsy: graph theoretical analysis of intracerebrally recorded mesial temporal lobe seizures. Clin. Neurophysiol. 118, 918-927. doi: 10.1016/j.clinph.2006.12.002

Ponten, S. C., Douw, L., Bartolomei, F., Reijneveld, J. C., and Stam, C. J. (2009). Indications for network regularization during absence seizures: weighted and unweighted graph theoretical analyses. Exp. Neurol. 217, 197-204. doi: 10.1016/j.expneurol.2009.02.001

Schindler, K., Leung, H., Elger, C. E., and Lehnertz, K. (2007). Assessing seizure dynamics by analysing the correlation structure of multichannel intracranial EEG. Brain 130, 65-77. doi: 10.1093/brain/awl304

Schindler, K. A., Bialonski, S., Horstmann, M. T., Elger, C. E., and Lehnertz, K. (2008). Evolving functional network properties and synchronizability during human epileptic seizures. Chaos 18, 033119. doi: 10.1063/1.2966112

Schoen, W., Chang, J. S., Lee, U., Bob, P., and Mashour, G. A. (2011). The temporal organization of functional brain connectivity is abnormal in schizophrenia but does not correlate with symptomatology. Conscious. Cogn. 20, 1050-1054. doi: 10.1016/j.concog.2010.05.009

Shinnar, S., Kang, H., Berg, A. T., Goldensohn, E. S., Hauser, W. A., and Moshe, S. L. (1994). EEG abnormalities in children with a first unprovoked seizure. Epilepsia 35, 471-476. doi: 10.1111/j.1528-1157.1994.tb02464.x

Smit, D. J., Boersma, M., Schnack, H. G., Micheloyannis, S., Boomsma, D. I., Hulshoff Pol, H. E., et al. (2012). The brain matures with stronger functional connectivity and decreased randomness of its network. PLoS ONE 7:e36896. doi: 10.1371/journal.pone.0036896

Smith, S. J. (2005). EEG in the diagnosis, classification, and management of patients with epilepsy. J. Neurol. Neurosurg. Psychiatry 76(Suppl. 2), ii2-7. doi: 10.1136/jnnp.2005.069245

Stam, C. J. (2010). Characterization of anatomical and functional connectivity in the brain: a complex networks perspective. Int. J. Psychophysiol. 77, 186-194. doi: 10.1016/j.ijpsycho.2010.06.024

Stam, C. J., De Haan, W., Daffertshofer, A., Jones, B. F., Manshanden, I., Van Cappellen Van Walsum, A. M., et al. (2009). Graph theoretical analysis of magnetoencephalographic functional connectivity in Alzheimer's disease. Brain 132, 213-224. doi: 10.1093/brain/awn262

Stam, C. J., Nolte, G., and Daffertshofer, A. (2007). Phase lag index: assessment of functional connectivity from multi channel EEG and MEG with diminished bias from common sources. Hum. Brain Mapp. 28, 1178-1193. doi: 10.1002/hbm.20346

Stam, C. J., and Van Straaten, E. C. (2012). The organization of physiological brain networks. Clin. Neurophysiol. 123, 1067-1087. doi: 10.1016/j.clinph.2012.01.011

Van Diessen, E., Diederen, S. J., Braun, K. P., Jansen, F. E., and Stam, C. J. (2013). Functional and structural brain networks in epilepsy: what have we learned? Epilepsia 54, 1855-1865. doi: 10.1111/epi.12350

Van Wijk, B. C., Stam, C. J., and Daffertshofer, A. (2010). Comparing brain networks of different size and connectivity density using graph theory. PLoS ONE 5:e13701. doi: 10.1371/journal.pone.0013701

Varela, F., Lachaux, J. P., Rodriguez, E., and Martinerie, J. (2001). The brainweb: phase synchronization and large-scale integration. Nat. Rev. Neurosci. 2, 229-239. doi: 10.1038/35067550

Von Stein, A., and Sarnthein, J. (2000). Different frequencies for different scales of cortical integration: from local gamma to long range alpha/theta synchronization. Int. J. Psychophysiol. 38, 301-313. doi: 10.1016/S0167-8760(00) 00172-0 
Watts, D. J., and Strogatz, S. H. (1998). Collective dynamics of "small-world" networks. Nature 393, 440-442. doi: 10.1038/30918

Wirrell, E. C. (2010). Prognostic significance of interictal epileptiform discharges in newly diagnosed seizure disorders. J. Clin. Neurophysiol. 27, 239-248. doi: 10.1097/WNP.0b013e3181ea4288

Conflict of Interest Statement: The authors declare that the research was conducted in the absence of any commercial or financial relationships that could be construed as a potential conflict of interest.

Received: 20 September 2013; accepted: 08 April 2014; published online: 29 April 2014.
Citation: van Diessen E, Otte WM, Braun KPJ, Stam CJ and Jansen FE (2014) Does sleep deprivation alter functional EEG networks in children with focal epilepsy? Front. Syst. Neurosci. 8:67. doi: 10.3389/fnsys.2014.00067

This article was submitted to the journal Frontiers in Systems Neuroscience.

Copyright (c) 2014 van Diessen, Otte, Braun, Stam and Jansen. This is an openaccess article distributed under the terms of the Creative Commons Attribution License (CC BY). The use, distribution or reproduction in other forums is permitted, provided the original author(s) or licensor are credited and that the original publication in this journal is cited, in accordance with accepted academic practice. No use, distribution or reproduction is permitted which does not comply with these terms. 